\title{
The importance of direct immunofluorescence in pemphigus herpetiformis diagnosis*
}

\author{
Paula Carolina Pessanha de Faria ${ }^{1}$ \\ Luna Azulay Abulafia ${ }^{1,2}$ \\ Flávia de Freire Cassia ${ }^{2}$
}

\author{
Camila Caberlon Cruz ${ }^{2}$ \\ Juan Manuel Pineiro Maceira ${ }^{3}$ \\ Paula Mota Medeiros ${ }^{4}$
}

DOI: http:/ /dx.doi.org/10.1590/abd1806-4841.20174510

\begin{abstract}
Pemphigus herpetiformis is an autoimmune bullous disease, that combines clinical features of dermatitis herpetiformis and linear IgA bullous dermatosis and immunological characteristics of pemphigus, which makes this disease peculiar and this diagnosis rarely suspected in the first evaluation of the patient. The reported case is of a patient with clinically bullous disease similar to dermatitis herpetiformis, whose multiple biopsies were inconclusive, and only after direct immunofluorescence with a pemphigus pattern (intraepidermal intercellular pattern) the confirmation of the diagnosis was possible.
\end{abstract}

Keywords: Skin diseases, vesiculobullous; Pemphigus; Fluorescent antibody technique, direct

\section{INTRODUCTION}

Described by Jablonska et al in 1975, pemphigus herpetiformis $(\mathrm{PH})$ is a rare pemphigus variant that associates the clinical features of dermatitis herpetiformis (DH) and linear IgA bullous dermatosis (LABD) with a heterogenous histology and direct immunofluorescence (DIF) similar to pemphigus - intercellular intraepidermal pattern.

PH has autoantibodies directed against desmoglein-1, less frequently against desmoglein-3 and rarely against desmocollins; sometimes no antigen is identified. ${ }^{1-4}$

Its incidence is of $6-7 \%$ among pemphigus patients, with no gender predilection, affecting the age group from 5 to 92 years. ${ }^{5,6}$ It manifests clinically as urticarial, annular plaques, with vesicles/ bullae in the periphery, located on the trunk and proximal limbs, associated to intense pruritus, with no mucosal involvement. ${ }^{1,5}$

\section{CASE REPORT}

Forty-one-year-old female patient, phototype IV, with recurrent, itchy, erythematous-edematous annular plaques, with overlying vesicles and crusts on the trunk and limbs for 3 years (Figures 1 and 2). The diagnostic considerations were of dermatitis herpetiformis and linear IgA bullous dermatosis. She was treated with antihistamines, emollients and gluten-free diet. Hepatitis, HIV and syphilis serologies were negative; blood count, liver and renal function tests were normal. Anti-endomysial, anti-transglutaminase and anti-gliadin antibodies were negative. Histopathology revealed mild acantholysis simulating spongiosis, exocytosis and perivascular inflammatory infiltrate in one of the biopsies, and acantholytic vesicle containing neutrophils and eosinophils in the other (Figures 3 and 4). DIF demonstrated intercellular IgG and C3 in the epidermis, in a pemphigus pattern, ruling out the previous suspi-

Work submitted on 26.02.2016

Approved by the Advisory Board and accepted for publication on 16.05.2016

* Study conducted at Universidade do Estado do Rio de Janeiro (UERJ) - Rio de Janeiro (RJ), Brazil.

Financial Support: None.

Conflict of Interests: None.

1 Dermatology Outpatient Clinic of the Department of Dermatology of the Universidade do Estado do Rio de Janeiro (UERJ) - Rio de Janeiro (RJ), Brazil.

2 Dermatology Outpatient Clinic of the Instituto de Dermatologia Professor Rubem David Azulay, Santa Casa da Misericórdia do Rio de Janeiro - Rio De Janeiro (RJ), Brazil.

3 Pigmented Lesions Outpatient Clinic of the Department of Dermatology of the Universidade do Estado do Rio de Janeiro (UERJ) - Rio de Janeiro (RJ), Brazil.

4 Dermatology Outpatient Clinic at Hospital do Açúcar de Alagoas do Centro Universitário Tiradentes, Maceió, Alagoas (AL), Brazil. 
cions of $\mathrm{DH}$, that would present with a deposit at the top of dermal papillae and LABD, that would present with a linear deposit of IgA along the basement membrane zone (Figure 5).

In face of the clinical features of herpetiform lesions and DIF in a pemphigus pattern, the conclusion was pemphigus herpetiformis. Treatment was commenced with dapsone, with partial improvement. However, at the dose of $100 \mathrm{mg}$ / day, the patient developed hemolytic anemia and the medication was replaced with prednisone $40 \mathrm{mg} /$ day, that provided complete clinical remission within a few days. To taper prednisone, we added mycophenolate mofetil 1000mg/day.

\section{DISCUSSION}

The patient had annular and circinate urticarial lesions, with small grouped vesicles and pustules, frequently in a herpeti-

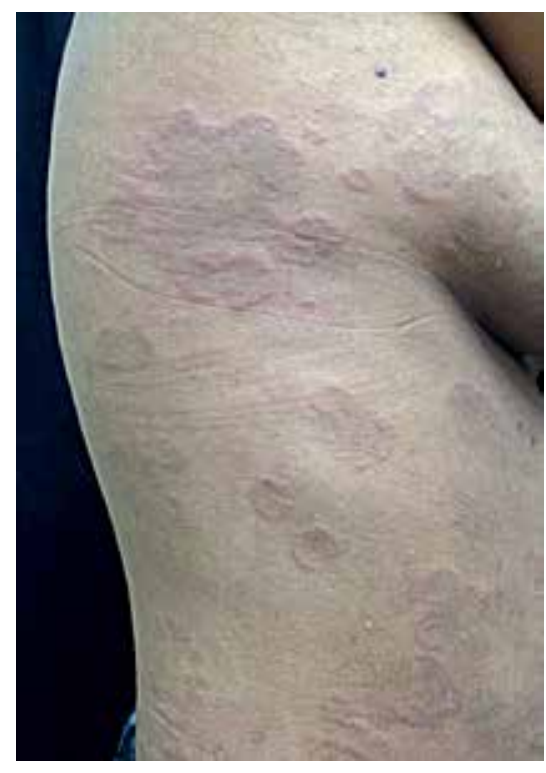

Figure 1:

Erythematous,

edematous annular

plaques with

centrifugal growth

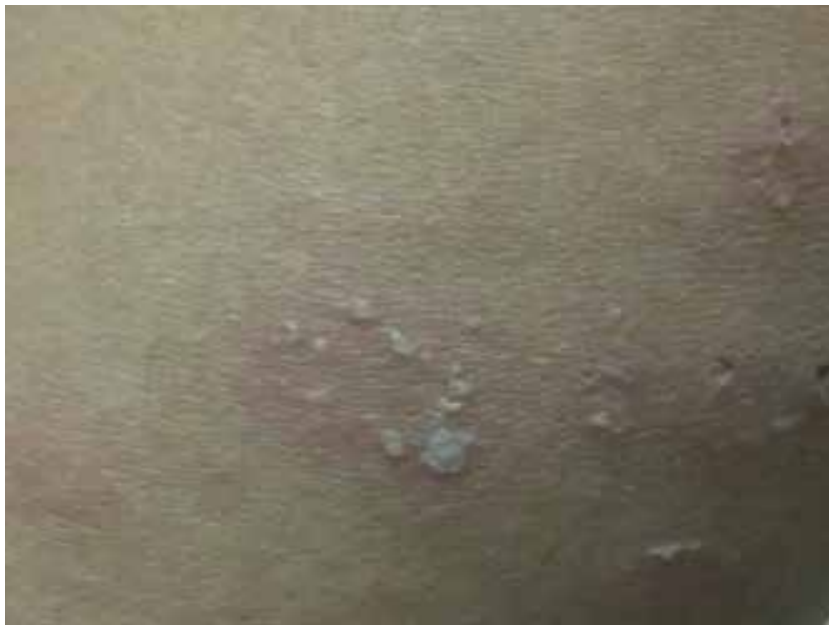

Figure 2: Erythematous, edematous plaques with overlying vesicles in a herpetiform pattern

An Bras Dermatol. 2017;92(5 Suppl 1):145-7. form pattern or in the periphery of the plaques, like a "string of pearls" (term frequently used for LABD), intensely pruritic. They were localized on the trunk and proximal extremities, as described by Jablonska et al. ${ }^{1}$

Histopathology is heterogenous, according to the clinical stage of the lesions, ranging from spongiosis with exocytosis to the typical or minimal acantholysis, more than one biopsy being sometimes needed in different phases in order to visualize the acantholysis, as with the reported patient. The inflammatory infiltrate in the biopsies of $\mathrm{PH}$ patients is predominantly composed of eosinophils (68\%), neutrophils (16\%) and mixed (16\%), according to Huhn et al. Peripheral eosinophilia can occur in some patients. ${ }^{5,7}$ In our case, four biopsies were performed in different departments. The first two showed spongiotic dermatitis with the formation of blisters, and the presence of neutrophils and eosinophils. In the third, there

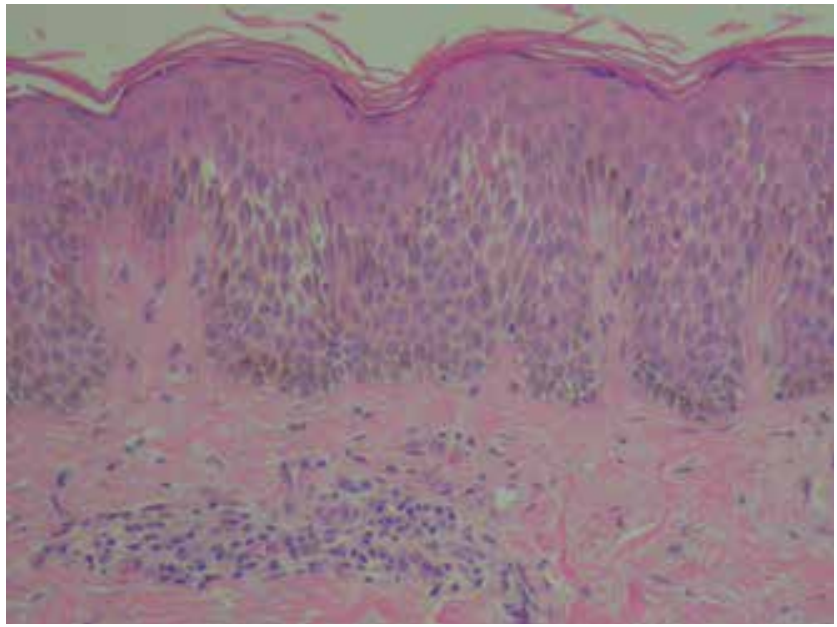

FIGURE 3: Exocytosis and mild acantholysis simulating spongiosis, besides predominantly mononuclear perivascular inflammatory infiltrate (Hematoxylin \& eosin, X100)

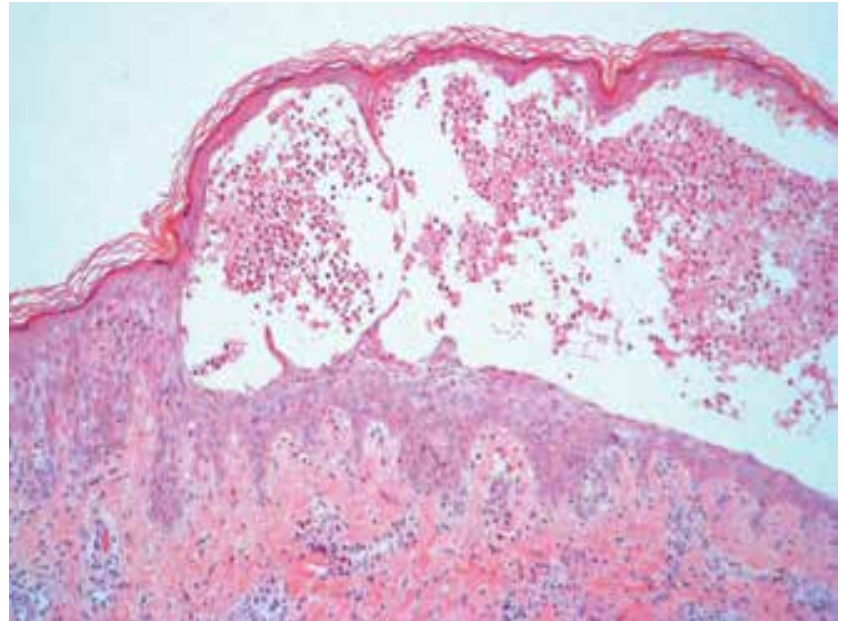

FIGURE 4: Intraepidermal acantholytic blister with numerous eosinophils, also seen in the adjacent epidermis (Hematoxylin \& eosin, X100) 


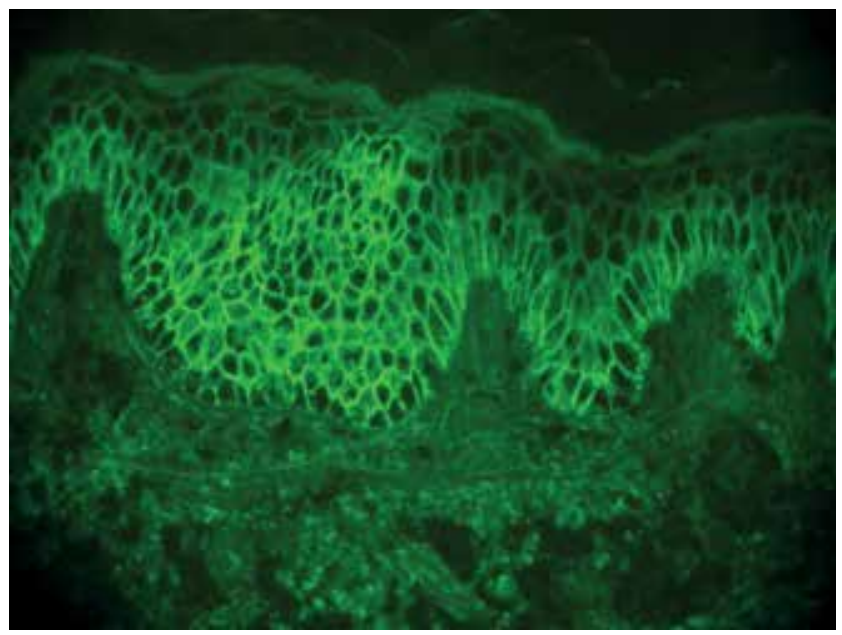

Figure 5: Deposition of IgG in an intercellular pattern all over the epidermis (IFD, X100)

was mild exocytosis and acantholysis, simulating spongiosis. In the fourth, an intraepidermal acantholytic blister was seen, containing multiple eosinophils, as already described. ${ }^{7}$

Perilesional skin DIF shows the presence of intercellular IgG and $\mathrm{C}_{3}$ in the epidermis, as in the reported case, characterizing it as a variant of pemphigus, crucial for the diagnosis. ${ }^{5}$

$\mathrm{PH}$ has a relatively benign course and good response to treatment with dapsone, the drug of choice, or low-dose systemic corticosteroids, such as in our patient. ${ }^{6}$ The use of immunosuppressants such as azathioprine and cyclophosphamide must be considered according to the severity of each case and as a way of avoiding the side effects of systemic corticosteroids. ${ }^{2,5,8}$ Mycophenolate mofetil $500 \mathrm{mg}$ twice daily was added with the intent to gradually taper the corticosteroid.

Some rare reports have demonstrated an association with lung, esophagus and prostate cancers, cutaneous angiosarcoma, infections (HIV), inflammatory conditions (systemic lupus erythematosus, psoriasis) and drugs. However, due to the rarity of this condition, it would be precocious to assume a paraneoplastic character of $\mathrm{PH}$, more studies being needed regarding this association. ${ }^{5,9}$

Recognizing $\mathrm{PH}$ is extremely important for the dermatologist, because the therapeutic approach differs from the other types of pemphigus, determining another course for the disease with improvement in the patient's quality of life.

Besides, it is important to highlight that since it can clinically mimic other bullous diseases (DH and LABD) and that the histopathology can be inconclusive (depending on the stage of the disease), the finding of epidermal intercellular IgG and C3 on DIF is extremely important for establishing a diagnosis. $]$

\section{REFERENCES}

1. Jablonska S, Chorzelski TP, Beutner EH, Chorzelska J. Herpetiform pemphigus, a variable pattern of pemphigus. Int J Dermatol. 1975;14:353-9.

2. Robinson ND, Hashimoto T, Amagai M, Chan LS. The new pemphigus variants. J Am Acad Dermatol. 1999 May:40(5 Pt 1):649-71.

3. Ishii $K$, Amagai M, Komai A, Ebihara T, Chorzelski TP, Jablonska $S$, et al. Desmoglein 1 and desmoglein 3 are the target autoantigens in herpetiform pemphigus. Arch Dermatol. 1999;135:943-7.

4. Miura T, Kawakami Y, Oyama N, Ohtsuka M, Suzuki Y, Ohyama B, et al. A case of pemphigus herpetiformis with absence of antibodies to desmogleins 1 and $3 . \mathrm{J}$ Eur Acad Dermatol Venereol. 2010;24:101-3.

5. Porro AM, Caetano $L$ de VN, Maehara $L$ de SN, Enokihara MM. Formas não clássicas de pênfigo: pênfigo herpetiforme, pênfigo por IgA, pênfigo paraneoplásico e pênfigo por IgG/lgA. An Bras Dermatol. 2014;89:96-106.

6. Kasperkiewicz M, Kowalewski C, Jabłońska S. Pemphigus herpetiformis: From first description until now. J Am Acad Dermatol. 2014;70:780-7.

7. Huhn KM, Tron VA, Nguyen N, Trotter MJ. Neutrophilic spongiosis in pemphigus herpetiformis. J Cutan Pathol. 1996;23:264-9.

8. Santi CG, Maruta CW, Aoki V, Sotto MN, Rivitti EA, Diaz LA. Pemphigus herpetiformis is a rare clinical expression of nonendemic pemphigus foliaceus, fogo selvagem, and pemphigus vulgaris. Cooperative Group on Fogo Selvagem Research. J Am Acad Dermatol. 1996;34:40-6.

9. Prado R, Brice SL, Fukuda S, Hashimoto T, Fujita M. Paraneoplastic pemphigus herpetiformis with IgG antibodies to desmoglein 3 and without mucosal lesions. Arch Dermatol. 2011;147:67-71. 